\title{
Planet formation in binary stars: the case of $\gamma$ Cephei
}

\author{
W. Kley ${ }^{1}$ and R. P. Nelson ${ }^{2}$ \\ 1 Institut für Astronomie und Astrophysik \& Kepler Center for Astro and Particle Physics, Universität Tübingen, \\ Auf der Morgenstelle 10, 72076 Tübingen, Germany \\ e-mail: wilhelm.kley@uni-tuebingen.de \\ 2 Astronomy Unit, School of Mathematical Sciences, Queen Mary, University of London, Mile End Road, London, E1 4NS, UK \\ e-mail: R.P.Nelson@qmw . ac . uk
}

Received 23 December 2007 / Accepted 7 May 2008

\section{ABSTRACT}

\begin{abstract}
Context. Over 30 planetary systems have been discovered to reside in binary stars. As some of the binary separations are smaller than 20 astronomical units (AU) the gravitational perturbation of the secondary star has a very strong influence on the planet formation process, as it truncates the protoplanetary disc, possibly shortens its lifetime, and stirs up the embedded planetesimals. Due to its small semi-major axis $(18.5 \mathrm{AU})$ and relatively large eccentricity $e=0.35$ the binary star $\gamma$ Cephei represents a particularly challenging example worthy of study in greater detail.

Aims. In the present study we model the orbital evolution and growth of embedded protoplanetary cores of about 30 earth masses in the putative protoplanetary disc surrounding the primary star in the $\gamma$ Cep system.

Methods. We assume coplanarity of the disc, binary, and planet and perform two-dimensional hydrodynamic simulations of embedded cores in a protoplanetary disc perturbed by a secondary companion. Before embedding the planet, the equilibrium structure of the disc for the observed binary parameters of $\gamma$ Cep is determined. We initiate the embedded planets in the disc on circular orbits with different initial distances from the primary.

Results. The presence of the eccentric secondary star perturbs the disc periodically and generates strong spiral arms at periapse that propagate toward the disc centre. The disc perturbations then weaken as the secondary approaches apoapse. The disc also becomes slightly eccentric $\left(e_{\mathrm{disc}} \approx 0.1-0.15\right)$, and displays a slow retrograde precession in the inertial frame. Embedded cores interact with the eccentric disc, and are periodically disturbed both by the strong spiral shocks and the eccentric binary. For all initial separations considered (from 2.5 to $3.5 \mathrm{AU}$ ) the cores migrate inward. In contrast, their eccentricity evolution depends primarily on the starting position in the disc. For initial semi-major axes $a_{\mathrm{p}} \gtrsim 2.7$ we find a strong increase in the planetary eccentricity despite the presence of inward migration. Only cores that are initially far from the disc outer edge $\left(a_{\mathrm{p}} \lesssim 2.7 \mathrm{AU}\right)$ have a bounded orbital eccentricity that converges, after mass accretion, roughly to the value of the planet observed in the $\gamma$ Cep system.

Conclusions. Even though a close binary system such as $\gamma$ Cep still presents a challenge to planet formation theory, we have shown that under the condition that protoplanetary cores can form at around $2.5 \mathrm{AU}$, it is possible to evolve and grow such a core to form a planet with a final configuration similar to what is observed.
\end{abstract}

Key words. methods: numerical - hydrodynamics - planets and satellites: formation - stars: binaries: general

\section{Introduction}

Among the known 280 extrasolar planets around main sequence stars, about 33 are known to reside in binary systems with a wide range of orbital separations (Eggenberger et al. 2004; Konacki 2005a; Mugrauer et al. 2005; Desidera \& Barbieri 2007). In all objects the planet orbits one of the two stars, while the second star acts as an external perturber to this system. These are the socalled S-type (stellar) orbits (Dvorak 1986). A truly "circumbinary planet" orbiting around a central binary star (a so-called P-type orbit) has not yet been detected.

In most of the systems the binary separation is large enough that the presence of a secondary may not have influenced the planet formation process around one of the stars too strongly. The subsequent long-term evolution of the planet in a binary may be shaped by the Kozai effect even for wider binaries (Wu \& Murray 2003; Takeda \& Rasio 2005; Wu et al. 2007). However, relatively short period binary orbits with binary separations of about $20 \mathrm{AU}$ and less have been found in a few systems, for example in G1 86 and notably in $\gamma$ Cephei. This raises questions concerning the formation and stability of planets in such systems. The reality of a planet in HD 188753 (Konacki 2005a), whose hypothetical existence caused some discussions about its origin in such a close binary system (Jang-Condell 2007; Portegies Zwart \& McMillan 2005; Pfahl 2005), has been put into question by newer observations (Eggenberger et al. 2007).

The mass-period and eccentricity-period distributions of extrasolar planets in binaries show interesting trends compared with planets around single stars, as presented in Eggenberger et al. (2004), and more recently in an updated version by Desidera \& Barbieri (2007). It is argued that for short planetary periods ( $\$ 40$ days) the distributions of mass and eccentricities in binaries are significantly different than for longer periods. Surprisingly, the closer-in planets in binaries tend to have small eccentricities very similar to or even smaller than in single stars. Additionally, there seems to be a large fraction of highmass planets with small orbital periods in binaries, that are not seen in single stars. However, presently the statistics are too small to allow definite conclusions. To improve on this deficiency in the amount of data, several new search programmes tailored in particular to planets in binary systems have been initiated (Konacki 2005b; Muterspaugh et al. 2005). Considering 
that observationally more and more planets are found to reside in binary stars, and may have orbital characteristics different from planets orbiting single stars, additional new calculations following the formation and evolution of such systems are urgently required.

Since planets form within protoplanetary discs which are tidally perturbed by the presence of a companion star one might expect consequences for the planet formation process. The most prominent effects are a truncation of the disc and an increase in the relative velocities of planetesimals. The influence a secondary has on the growth process through collisions from planetesimals to planetary embryos was studied first through 3-body calculation by Heppenheimer $(1974,1978)$ and later by Whitmire et al. (1998). Their results implies that for a solar mass star the minimum distance to an orbiting companion (periastron), such that planetesimals at $1 \mathrm{AU}$ can grow to larger bodies, must always be higher than about 16 AU. The critical semi-major axis scales only weakly with distance from the star. In the last years these small number studies have been extended to full $\mathrm{N}$-body simulations including several hundred planetesimals. These typically utilise a modern N-body integrator adapted for binary stars (e.g. Chambers et al. 2002), allow for direct collisions and sticking (Lissauer et al. 2004), and also gas drag (Turrini et al. 2005). For binary parameters such as $\alpha$ Cen terrestrial planet formation from an initial sea of planetesimals can proceed similar to the Sun-Jupiter system (Lissauer et al. 2004).

Another path of studying the formation of planets in binary stars was taken by Nelson (2000), who studied the interaction of two binary stars each of which is surrounded by its own circumstellar disc. The two discs are both modelled hydrodynamically, and during each binary orbit they are strongly perturbed at the time of periapse. Nelson (2000) argues that due to the periodic heating of the disc planet formation becomes more unlikely. On the one hand, the temperatures in the disc during periapse become so high that condensation of solid material is reduced, while on the other hand the increased temperatures in the spiral arms hinders the onset of gravitational instability. However, Boss $(1998,2006)$ has argued that in some cases the presence of the secondary may even induce planet formation through gravitational instability.

The dynamical evolution of Jovian protoplanets in circumbinary discs (P-type orbits) has been investigated by Nelson (2003) and Pierens \& Nelson (2008a), studies which were extended recently to include low mass protoplanets (Pierens \& Nelson 2007, 2008b).

As of today there is only one rather preliminary study (Kley 2000) dealing directly with the dynamical evolution of embedded protoplanets in external binary stellar systems. In that study a Jupiter mass planet was evolved in a protoplanetary disc for a range of binary separations $a_{\text {bin }}=50-100$ AU and a given binary eccentricity $e_{\text {bin }}=0.5$. In the binary environment the planet experienced a faster growth and migration with respect to the unperturbed case. However, the simulations were rather unrealistic in the sense that the planet was not grown in the disc from an embryo to its final size, but rather placed 'ready made' into an otherwise unperturbed disc.

In this contribution we would like to improve on these models, and in a future publication we will present our results of new simulations following the evolution and growth of planetesimals in the protoplanetary disc in a binary system. In this present study we consider the particular system $\gamma$ Cep because this is one of the tighter systems, and thus places rather strong constraints on the possible planet formation scenarios. For these reasons, this system has attracted much attention in past years. Several studies looked at the stability and/or the possibility of (additional) habitable planets in the system (Dvorak et al. 2004; Turrini et al. 2004; Haghighipour 2006; Verrier \& Evans 2006). Finally, the possibility to grow protoplanets from very small planetesimals in $\gamma$ Cep has been studied by Thébault et al. (2004), who placed km-sized planetesimals in a protoplanetary disc including gas drag and gravitational disturbances from the secondary and analysed the relative impact velocity. For a sufficient amount of gas present, the aerodynamic drag between gas and particle leads to periastron alignment such that that runaway growth is possible inside of $2.5 \mathrm{AU}$ from the primary star. A more extended study of this process for various dynamical parameter of the binary has been performed by Thébault et al. (2006). Recently Kley \& Nelson (2007) and Paardekooper et al. (2008) improved again on these models by considering the evolution of a swarm of planetesimals embedded in a hydrodynamically evolving disc.

In the present work we focus on $\gamma$ Cep and we assume, along the work of Thébault et al. (2004), that protoplanetary cores of a few earth masses have successfully formed inside the disc and follow the subsequent dynamical evolution and mass growth. We focus here on the later phase of gas accretion only and will present additional new results on planetesimal formation in a forthcoming paper (see also Kley \& Nelson 2007). We perform full numerical, hydrodynamical evolutions of protoplanetary discs for binary parameters typical for $\gamma$ Cep, analyse the equilibrium configuration of the disc, and study the subsequent evolution of embedded protoplanets.

This paper is organised as follows: in Sect. 2 we describe the model set-up, and in Sect. 3 we present results of disc evolution due to the binary but in the absence of the embedded planet. In Sect. 4 we present results of simulations with planets embedded in a protoplanetary disc perturbed by a binary companion. Finally, we draw our conclusions in Sect. 5.

\section{Model setup}

In modelling the putative early evolution of the $\gamma$ Cep system, we assume that the protoplanetary disc is in an S-type configuration and orbits around the more massive primary star. To clarify the primary effects of planetary growth and evolution in binaries, we avoid complex three-dimensional effects and assume that the whole system is coplanar, i.e. the orbits of the disc, the binary and the planet are all lying in one plane and the angular momenta are all aligned. To further simplify the calculations we treat the disc in a two-dimensional approximation, neglect any vertical extension and work with the vertically integrated equations. This approximation is often made for accretion discs and applies whenever the vertical thickness $H$ (pressure scale height) of the disc is small compared to the radial distance $r$ from the central star, i.e. $h \equiv H / r \ll 1$.

We use cylindrical coordinates $(r, \varphi, z)$, where the the origin of the coordinate system lies in the centre of the primary star at $r=0, z=0$. The evolution of all objects (stars, disc and planet) takes place in the $z=0$ plane. As the stars orbit each other, the coordinate system is non-inertial and the indirect terms have to be included in the equations of motion.

Accretion discs are most-likely driven by magnetohydrodynamical turbulence which we model here in a simplified manner through a viscous stress tensor model. For the kinematic viscosity coefficient $v$ we assume a standard $\alpha$-prescription with $v=\alpha c_{\mathrm{S}} H$, where $\alpha$ is a constant, and $c_{\mathrm{S}}$ is the local sound speed 
given by $c_{\mathrm{s}}(r)=h u_{\text {kep }}(r)$ where $u_{\text {kep }}(r)$ is the Keplerian velocity of the accretion disc around the primary star.

The basic hydrodynamic equations (mass and momentum conservation) describing the time evolution of such a viscous two-dimensional disc with embedded planets have been stated frequently and are not repeated here (see Kley 1999).

The dynamical effects of the companion star will lead to a disc structure which is different from that which exists around a single star. To account for this restructuring of the disc we first model the disc evolution in the binary system alone without any embedded planet. As will be shown below this initialisation phase takes about 100 binary orbits. Only after this time the disc has relaxed (on average) to its new equilibrium and then the planets are inserted into the models.

\subsection{Initial conditions}

The two-dimensional $(r-\varphi)$ computational domain consists of a complete ring $\left(\varphi_{\min }=0, \varphi_{\max }=2 \pi\right)$ of the protoplanetary disc that radially extends from $r_{\min }=0.50 \mathrm{AU}$ and $r_{\max }=8.0 \mathrm{AU}$. The computational domain is covered by $300 \times 300\left(N_{\mathrm{r}} \times N_{\varphi}\right)$ grid cells, that are spaced equidistant in both radius and azimuth.

The initial hydrodynamic structure of the disc (density, temperature, velocity) is axisymmetric with respect to the location of the primary star. The surface density has the profile $\Sigma(r)=\Sigma_{0} r^{-1 / 2}$ over the entire radial domain, where $\Sigma_{0}$ is chosen such that the total mass in the computational domain (within $r_{\min }$ and $r_{\max }$ ) equals $1.75 \times 10^{-3} M_{\odot}$ which implies $\Sigma_{0}=1.89 \times 10^{-5} M_{\odot} / \mathrm{AU}^{2}$. The initial velocity is pure Keplerian rotation $\left(u_{\mathrm{r}}=0, u_{\varphi}=\Omega_{K} r=\left(G M_{*} / r\right)^{1 / 2}\right)$, and the temperature stratification is always given by $T(r) \propto r^{-1}$ which follows from the assumed constant vertical height $h=H / r=0.05$. For these locally isothermal models the temperature profile is left unchanged at its initial state throughout the computations, i.e. no energy equation is solved.

\subsection{Boundary conditions}

The boundary conditions are chosen such that material may escape through the radial boundaries. At the outer boundary $\left(r_{\max }\right)$ we impose a so called zero-gradient outflow condition, where the velocity is extrapolated when directed outward and set to zero when directed inward in the last active gridcell inside $r_{\max }$. For all other variables zero gradients are assumed. During periastron when large spiral arms may extend beyond $r_{\max }$ this condition will allow material to leave the system freely and will help to reduce numerical artifacts. At the inner boundary we set a viscous outflow condition where the material may flow through $r_{\text {min }}$ with the local (azimuthally averaged) viscous inflow referring to an accretion disc in equilibrium,

$u_{\mathrm{r}}\left(r_{\min }\right)=-\frac{3}{2} \frac{\bar{v}}{r_{\min }}$,

where $\bar{v}$ denotes the azimuthally averaged viscosity at $r_{\min }$. We experimented with an outflow inner boundary similar to that used at $r_{\max }$ but found that in such a case the induced ellipticity of the disc (by the eccentric binary) will cause strong outflow of disc material in its periastron phase which in turn produces a large eccentric inner hole of the disc which is a numerical artefact. Our chosen inner boundary allows for accretion onto the star and additionally mimics the expected nearly circular flow in the inner regions of the disc close to the primary. No matter is allowed to flow back into the system and the mass of the disc will slowly decline.
Table 1. Orbital data of the binary $\gamma$ Cep and its planet as given by Hatzes et al. (2003), which have been used for the simulations.

\begin{tabular}{ccccccc}
\hline \hline$M_{1}$ & $M_{2}$ & $a$ & $e$ & $M_{\mathrm{p}}$ & $a_{\mathrm{p}}$ & $e_{\mathrm{p}}$ \\
{$\left[M_{\odot}\right]$} & {$\left[M_{\odot}\right]$} & {$[\mathrm{AU}]$} & & {$\left[M_{\mathrm{Jup}}\right]$} & {$[\mathrm{AU}]$} & \\
\hline 1.59 & 0.378 & 18.5 & 0.36 & 1.70 & 2.13 & 0.20 \\
\hline
\end{tabular}

\subsection{Physical parameters}

In the majority of our our numerical simulations we choose to model a specific system where the orbital elements of the binary have been chosen to match the system $\gamma$ Cep quite closely. The data for this system have been taken from Hatzes et al. (2003) which do not include the most recent observational improvements (Neuhäuser et al. 2007). These newest refinements primarily concern the mass of the primary and should not alter our main conclusions at all. The present studies are supposed to demonstrate the principle physical effects occurring in close binaries rather than trying to achieve a perfect match with all the observations of this system. For the runs we choose a binary with $M_{1}=1.59 M_{\odot}, M_{2}=0.38 M_{\odot}, a_{\text {bin }}=18.5 \mathrm{AU}$ and $e_{\text {bin }}=0.36$ (see Table 1 ), which translates into a binary period of $P=56.7$ yrs. For the viscosity we use a typical value for the effective viscosity in a protoplanetary disc, i.e. $\alpha=5 \times 10^{-3}$, and a locally isothermal disc with $h=H / r=0.05$.

As mentioned above, the initial total disc mass in the system is $1.75 \times 10^{-3} M_{\odot}$. Due to the presence of the secondary the disc will be truncated to a smaller outer radius and the radial density stratification will adjust to a new equilibrium configuration. At the same time mass will be lost from the system due to possible outflow through the (open) boundaries. To ensure a somewhat uniform initial configuration for the planets we rescale the disc mass to a given mass before inserting the planets.

\subsection{A few remarks on numerical issues}

To substantiate our results we use two different codes for our calculations, RH2D (Kley 1999, 1989) and NIRVANA (Nelson et al. 2000; Ziegler \& Yorke 1997), both of which have been utilised frequently in embedded planet problems. The numerical method used in both codes is a staggered mesh, spatially second order finite difference method based, where advection is based on the monotonic transport algorithm (van Leer 1977). Due to operatorsplitting the codes are semi-second order in time. The computational details of RH2D which can be used in different coordinate systems have been described in general in Kley (1989), and specifically for planet calculations in Kley (1999). The algorithmic details of the NIRVANA code have been described in Ziegler (1998). In all of our simulations we utilise a non-rotating coordinate frame centred on the primary.

In calculating the gravitational effect of the planet we use a smoothed potential of the form

$\Phi_{\mathrm{P}}=-\frac{G M_{\mathrm{p}}}{\sqrt{s^{2}+\epsilon^{2}}}$

where $s$ is the distance from the planet to the (grid-)point under consideration. For the smoothing length of the potential we choose $\epsilon=0.6 R_{\text {Hill }}$, where

$R_{\mathrm{Hill}}=r_{\mathrm{P}}\left(\frac{q}{3}\right)^{(1 / 3)}$.

Here $r_{\mathrm{P}}$ denotes the momentary distance of the planet from the star, i.e. the size of the Hill radius scales with the distance from 
the star and not the semi-major axis. This distinction is important for calculating the torques in the case of eccentric orbits.

The smoothing length $\epsilon$ is proportional to the local scale height $H$ of the disc to account for the vertical extent of real discs. The chosen value of $0.6 R_{\text {Hill }}$ gives a reasonably good agreement between the linear torques of a three-dimensional disc and the two-dimensional flat-disc approximation used here (Tanaka et al. 2002). In a recent study of the dynamical evolution of eccentric low mass planets we have found a good agreement for the migration and eccentricity damping rate in $2 \mathrm{D}$ and $3 \mathrm{D}$ discs for this value of $\epsilon$ (Cresswell et al. 2007).

To model possible mass accretion onto the protoplanet we take out mass within a given radius $r_{\mathrm{acc}}$ around the planet at a prescribed rate. Whenever the centre of a gridcell is closer than $r_{\text {acc }}=1 / 2 R_{\text {Hill }}$ we reduce the density in that cell by a factor $1-$ $f_{\text {acc }} \Delta t$, where $\Delta t$ the the actual time-step of the computation and $f_{\text {acc }}$ is a model dependent reduction factor. The rate is doubled whenever the distance is smaller than $1 / 4 r_{\text {Hill }}$. For details see Kley (1999).

The viscous terms, including all necessary tensor components, are treated explicitly. To ensure stability in the outer parts of the disc where we expect stronger gradients in the flow due to the perturbations by the secondary, an artificial bulk viscosity has been added, with a coefficient $C_{\text {art }}=1.0$. For the detailed formulation of the viscosity related issues and tests, see Kley (1999).

To avoid excessively low densities in the outer part of the computational domain we have found it preferable to work with a density floor, where the density cannot fall below a specified minimum value $\Sigma_{\min }$. For our purpose we use a value of $\Sigma_{\min }=$ $10^{-6}$ in dimensionless units, where the initial density is of $O(1)$ at $r=1.0$.

When calculating the gravitational forces of the disc acting on the planet numerically for a given grid resolution a cutoff radius $r_{t}$ is necessary in addition to the smoothing of the potential. This cutoff reduces the contribution of material in the direct vicinity of the planet which lies inside the planetary Roche-lobe. This material is bound to the planet and typically cannot exert any forces on it. Here we use a cutoff of $r_{t}=0.8 R_{\text {Hill }}$ for most runs which is smoothed at $r_{t}$ using a Fermi-function with a width of $0.1 r_{t}$. The role of the cutoff radius $r_{t}$ in estimating the torque on the planet has been analysed in more detail by Crida et al. (2008). For the range $0.6 R_{\text {Hill }} \leq r_{t} \leq 1.0 R_{\text {Hill }}$ the obtained results agree very well with each other, see also Sect. 4.1 below.

The planet evolution and the orbital motion of the binary proceed on time scales much longer than the typical dynamical time scale of the disc, and consequently very many time-steps need to be calculated just in one simulation. To allow for parameter studies we have increased the performance of the runs by implementing the FARGO-algorithm in our codes, which is particularly designed to model differentially rotating flows efficiently (Masset 2000). For our chosen radial range and grid resolution we find a speed-up factor of about 7.5 over the standard case. Then, applying a Courant number of 0.75, still about 160000 time-steps are required for only 10 binary orbits for our radial range and a standard resolution of $300 \times 300$. We checked the accuracy of the FARGO implementation against several test cases.

\section{Disc evolution without an embedded planet}

When studying the formation of planets in a protoplanetary disc in the presence of a secondary star it is necessary and illustrative to first investigate the structure and evolution of the accretion disc subject to the influence of the binary system only. Hence,

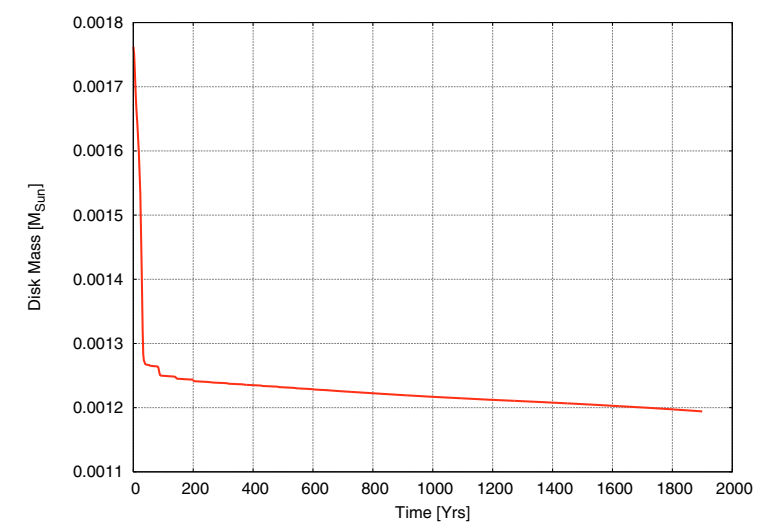

Fig. 1. Time evolution of the total disc mass in units of the $M_{\odot}$ vs. time in yrs for no embedded planet. Note that one binary orbit refers to about 57 yrs.
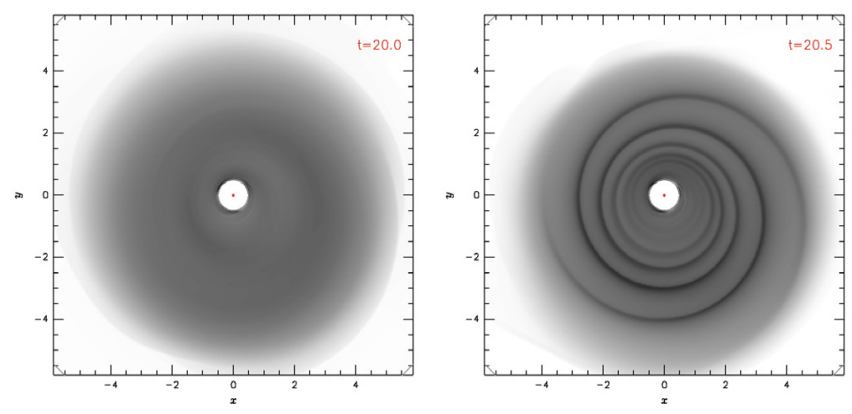

Fig. 2. Grayscale plot of the two-dimensional density distribution of the circumstellar disc around the primary at two different orbital phases of the binary. Left shortly after apocentre at about 20 binary orbits, and right shortly after closest approach (pericentre).

in this section we follow the evolution of a disc around the primary which is perturbed by the secondary without an embedded planet. As the disc evolves slowly from its initial axisymmetric state into a new quasi-equilibrium which is quite different from the unperturbed initial state, we have first to relax the system into this new equilibrium, before adding a planetary embryo at a later time. In the following we describe in more detail this restructuring process of the disc and the change in the binary elements due the finite disc mass.

\subsection{The structure of the disc}

The presence of an eccentric secondary star leads to a strong periodic disturbance of the disc whenever it is at periastron. Then two strong tidal spiral arms (shock waves) are excited in the disc which wind themselves all the way down to the centre. At the outer edge of the disc the perturbation by the companion star carries material beyond the outer boundary of the computational domain which is then lost from the system. During the very first periapse phase about $30 \%$ of the initial disc mass is lost from the system, and very little during the following passages, as show in Fig. 1. In between the periapses the disc settles down and becomes more circular again. This effect is illustrated in Fig. 2 where we display the surface density $\Sigma$ of the disc in gray scale at 2 different times in the early evolution of the disc. In the left panel the nearly circular state at the binary apoastron is displayed while the right panel shows the situation just after periastron. These findings are very similarly to those found by Nelson (2000) in his study of the binary system L1551. 

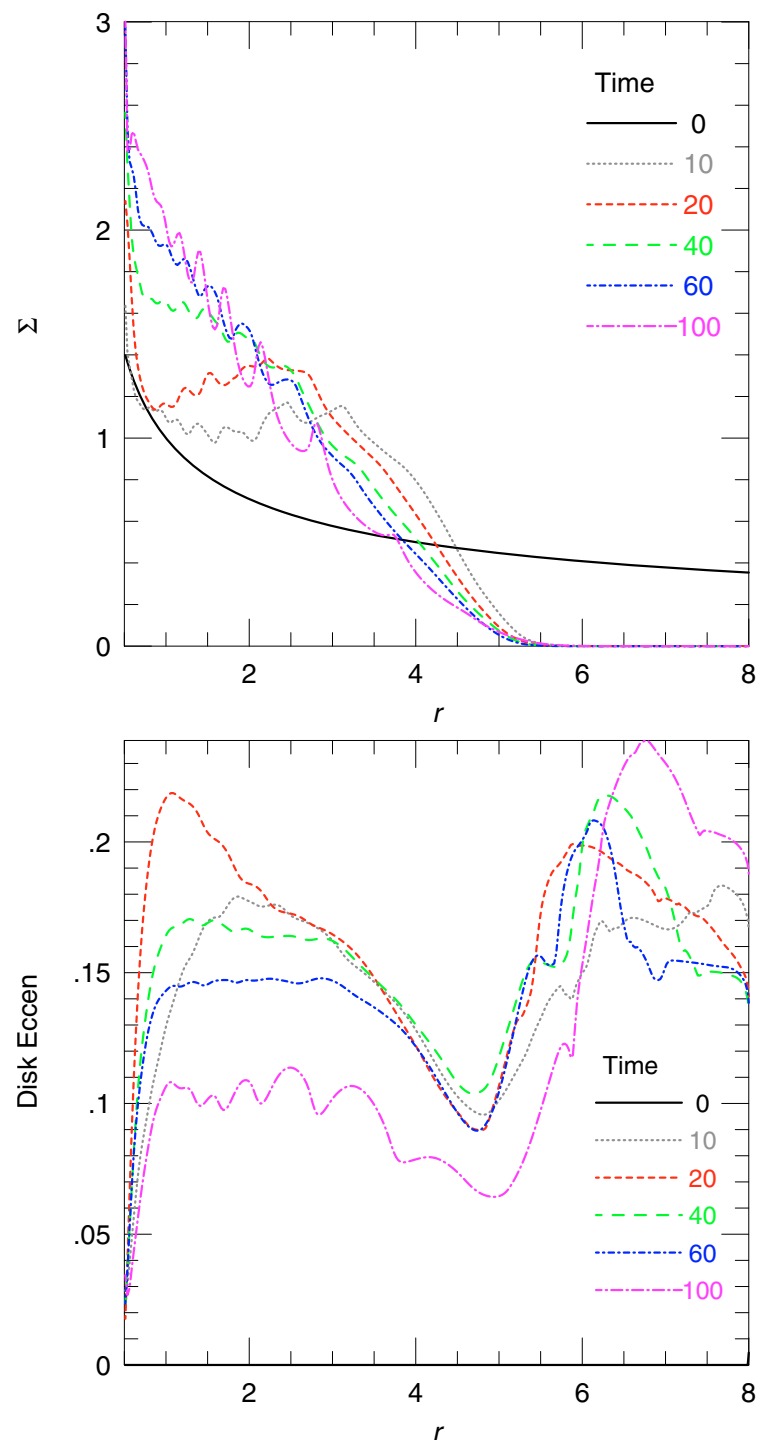

Fig. 3. Radial dependence of the azimuthally averaged surface density (top) and the eccentricity (bottom) of the circumstellar disc around the primary in the presence of the secondary at different times. Time is given units of the binary orbit, radial distance in $\mathrm{AU}$, and the density in dimensionless units.

Already the very first close approaches with the binary lead to a clear truncation of the disc at around $5 \mathrm{AU}$, as is visible in left panel of Fig. 3 for the curve at $t=10$ binary orbits. Slowly the whole disc structure rearranges and equilibrates at around $t=50$ where it shows a much steeper (nearly linear) density slope than in the initial state. The time scale for this equilibration process depends on the magnitude of the disc viscosity and shock dissipation. For relatively cool discs and small viscosities we expect that the equilibrium profile is primarily determined by the same processes, i.e. gravitational action of the binary and shock dissipation. The eccentricity of the disc in the final state of the disc varies approximately between 0.1 and 0.16 depending on the position of the binary in its orbit as shown in the bottom panel of Fig. 3. In the outer parts of the disc beyond $r \approx 5 \mathrm{AU}$, where the density is very low, the disc eccentricity is measured to be larger due to the proximity to the secondary star.

The overall (mass averaged) eccentricity of the disc displays oscillations on longer time scales of about 17 binary orbits around the mean value of 0.12 (left panel of Fig. 4). The disc eccentricity $e_{\mathrm{disc}}(r)$ in that plot has been obtained by calculating
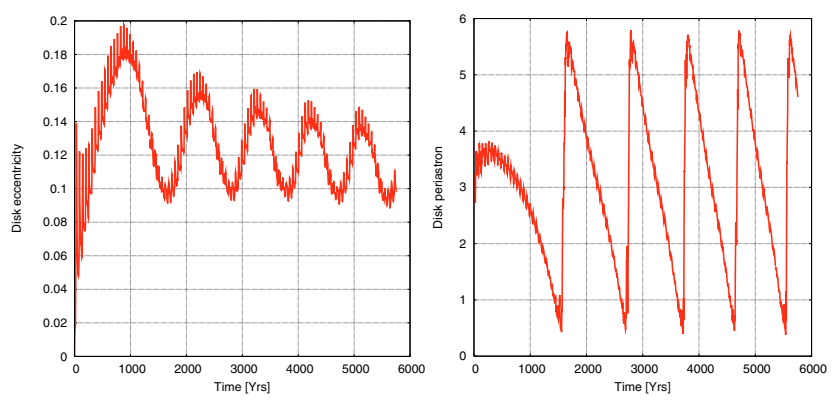

Fig. 4. The evolution of the global (density weighted) averaged disc eccentricity (left) and the position angle of the disc's periapse (right).

the eccentricity of each disc element, as if in a two body motion with the primary star, and then averaged azimuthally over the respective annulus. The superposed spikes are caused by the individual periapses of each orbit, during which the whole disc is perturbed by the strong spiral arms. At the same time the disc as a whole precesses, as is shown in the right panel of Fig. 4. This coherent slow precession is retrograde with a pattern speed much smaller than the orbital period of the disc material around the star. This interesting global disc phenomenon appears to be related to the induced precession of accretion discs in close binary systems such as cataclysmic variable stars which occurs even in the case of circular binaries (Lubow 1991). Very similar results have recently been found by Paardekooper et al. (2008), see also Kley \& Nelson (2007). In test simulations using circular and non-circular binaries we have also verified that eccentric discs occur in the circular case as well, and in recent simulations Paardekooper et al. (2008) have also demonstrated (for $\gamma$ Cep parameter) that in the limit of circular orbits the mechanism that causes the instability is indeed the 3:1 eccentric Lindblad resonance (Lubow 1991). In a related study we have analysed in detail the influence of numerical parameter on the occurrence and properties of eccentric discs in binaries and we refer the reader to that paper for more details (Kley, Papaloizou \& Ogilvie, to be submitted). For the highly truncated disc in this study we expect the averaged eccentricity to be relatively robust against numerical variations. Test simulations of this initial equilibration phase of the disc with different grid resolutions resulted in very similar values of the disc eccentricity. For the typical values of disc viscosity and temperature used by us we expect these values for the disc eccentricity, an expectation which is confirmed by Paardekooper et al. (2008) who find find very similar results. Concerning the evolution of embedded protoplanets in these discs it appears that the effect of the periodic disturbance of the binary may play a more important role than the eccentricity of the disc.

The direction of the precession (prograde or retrograde with respect to the disc and binary motion) is influenced by pressure effects in the disc. For our chosen value of $H / r=0.05$ we do indeed expect a retrograde precession. Similar behaviour has also been demonstrated for discs with free eccentricity (Papaloizou 2005).

\subsection{The orbital elements of the binary}

In the previous section we have seen that the gravitational torques of the binary lead to truncation of the disc and rearrangement of the material within. In turn, we expect a change in the orbital elements of the binary. 

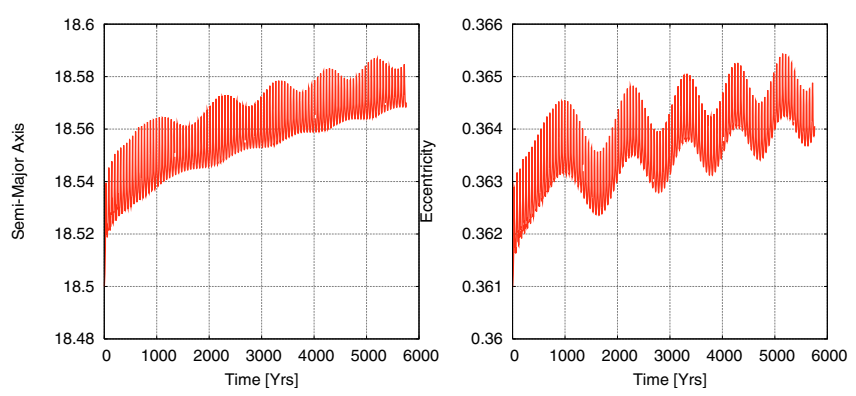

Fig. 5. The evolution of the binary elements due to the interaction with the circumstellar disc around the primary star, without an embedded planet. One binary orbit refers to approximately 57 yrs. Left: $a_{\text {bin }}(t)$; right: $e_{\mathrm{bin}}(t)$.

To estimate theoretically the magnitude of the back-reaction a circumstellar disc has on the orbital elements of the binary we assume an idealised system consisting of a binary system and a ring-like mass distribution orbiting star 1 with mass $m_{\text {ring, }}$, at a distance ( $\delta$-function) of $r_{\text {ring }}$. The energy $E_{\text {bin }}$ and angular momentum $L_{\text {bin }}$ of the binary is given by

$E_{\text {bin }}=-\frac{G M \mu}{2 a_{\text {bin }}}, \quad L_{\text {bin }}=\mu\left(G M a_{\text {bin }}\left(1-e_{\text {bin }}^{2}\right)\right)^{1 / 2}$,

and the corresponding quantities of the ring are

$E_{\text {ring }}=-\frac{G M_{1} m_{\text {disc }}}{2 r_{\text {ring }}}, \quad L_{\text {ring }}=m_{\text {ring }}\left(G M_{1} r_{\text {ring }}\right)^{1 / 2}$,

where $M=M_{1}+M_{2}$ is the total mass of the two stars and $\mu=M_{1} M_{2} / M$ is the reduced mass. Now, suppose that the ring is shifted from its initial position $r_{\text {ring }}^{\alpha}$ to a smaller radius $r_{\text {ring }}^{\beta}$ keeping all its mass. This radius change mimics the truncation of disc by the binary. Through this process the ring's energy and angular momentum are reduced from $E_{\text {ring }}^{\alpha}$ and $L_{\text {ring }}^{\alpha}$ to $E_{\text {ring }}^{\beta}$ and $L_{\text {ring }}^{\beta}$. By conservation of total energy and angular momentum

$E=E_{\text {ring }}+E_{\text {bin }} \quad L=L_{\text {ring }}+L_{\text {bin }}$.

We can calculate the corresponding change in the orbital elements of the binary from $E_{\text {bin }}^{\alpha}$ and $L_{\text {bin }}^{\alpha}$ to $E_{\text {bin }}^{\beta}$ and $L_{\text {bin }}^{\beta}$. For the binary parameter masses $M_{1}=1.6 M_{\odot}, M_{2} \stackrel{\text { bin }}{=} 0.4 M_{\odot}$ with initial orbital elements $a_{\text {bin }}^{\alpha}=18.5 \mathrm{AU}$ and $e_{\text {bin }}^{\alpha}=0.36$ we find for the shift of a ring with $m_{\text {ring }}=4 \times 10^{-3} M_{\odot}$ and initial radius $r_{\text {ring }}^{\alpha}=4.0 \mathrm{AU}$ to a final radius of $r_{\text {ring }}^{\beta}=2.0 \mathrm{AU}$ that the binary elements change to $a_{\text {bin }}^{\beta}=19.4 \mathrm{AU}$ and $e_{\text {bin }}^{\beta}=0.41$. A quite substantial change considering the smallness of the ring's mass in comparison to the stellar masses. But the closeness of the ring to the which is primary allows it to gain a substantial amount of binding energy. The calculation is approximate in the sense that the energy and angular momentum of the ring are calculated with respect to the non-inertial coordinate frame centred on the primary.

We can now compare this estimate with the previous hydrodynamical simulations, and plot in Fig. 5 the evolution of $a_{\text {bin }}$ and $e_{\text {bin }}$ for about the first 100 binary periods without a planet included. As demonstrated above, the binary expands as it gains energy from the truncated disc and increases its eccentricity. The increase in $e_{\text {bin }}$ does not lead to a decrease in the angular momentum however, since it increases its separation, see Eq. (4). Whenever the binary is near periastron the gravitational interaction with the disc is maximal which results in the strong periodic spikes in the binary elements. The change in the orbital elements of the binary is somewhat smaller than the estimated values because i) the disc mass is smaller in the hydrodynamic calculation and ii) disc mass and angular momentum are stripped off by the secondary and are lost through the outer boundary of the computational domain. The loss through the (open) inner boundary of the disc is only marginal.

\section{Evolution with an embedded planet}

In the previous section we have analysed the change of the orbital elements of the binary caused by the mass rearrangement in the disc. Now we turn to the embedded planet in the disc, which is inserted into the disc after an equilibration time of 100 binary orbits (nearly $6000 \mathrm{yrs}$ ). This rather time consuming procedure to generate the initial state is necessary to obtain realistic initial conditions for the growing protoplanet. At the time of insertion of the planet the remaining disc mass is rescaled (through $\Sigma_{0}$ ) to contain $3 M_{\text {Jup }}$ within the computational domain, while keeping its two-dimensional distribution unchanged. To follow the growth and evolution from planetary cores to massive planets the mass of the inserted protoplanet is $36 M_{\text {Earth }}$ orbiting the primary star. This mass was chosen as it corresponds closely to the mass above which forming gas giant planets undergo the final phase of rapid gas accretion (Papaloizou \& Nelson 2005). The typical starting values of the semi-major axis and eccentricity are $a_{\mathrm{p}}=2.5 \mathrm{AU}$ and $e_{\mathrm{p}}=0.0$.

\subsection{Exploratory models and numerical tests}

After inserting the protoplanet on a circular orbit at $2.5 \mathrm{AU}$ we expect that its orbital elements will change due to the gravitational influence of the binary and the distorted disc. To differentiate the different contributions we decided to check the origin of the dynamical behaviour, through a variation of physical conditions. The standard model resembles the true physical situation where the planet feels the full influence of the binary and the disc which is perturbed by the binary. In the other set-ups we switch the various contributions on and off. Avoiding additional complications in this analysis, the protoplanet is not allowed to accrete here $\left(f_{\text {acc }}=0\right)$ and its mass remains fixed at $36 M_{\text {Earth }}$.

The results are displayed in Fig. 6, where the semi-major axis and eccentricity of the planet are shown for four different set-ups. The standard model (h26, dark solid line) refers to the full model (including binary and disc) as just described, in model (h26a, dashed bright line) the mass of the secondary has been switched off to test its influence, and the planet evolves in the initially eccentric disc which becomes more circular during the evolution because of the absence of the secondary. In model (h26b, dark dotted line) in addition to having no secondary the density in the disc has been azimuthally symmetrized keeping the radial distribution intact. Hence, this model suits as a reference of what happens in the single star case. In the last model (h26c, fine dotted line) the secondary is present but the disc mass has been reduced, such that effectively only a 3-body problem is solved. The curves with the periodic bumps in Fig. 6 refer to the cases (h26, h26c) including the secondary while the smoother curves describe the situation where the secondary star has been excluded (h26a, h26b). The bumps occur with the binary period and indicate the "kick" the planet experiences due to the interaction with the spiral arms in the disc and the direct interaction with the binary companion when it is at periastron. The results show that the main contribution to the initial growth of planetary eccentricity $e_{\mathrm{p}}$ is in fact the eccentric disc. The eccentricity $e_{\mathrm{p}}$ 

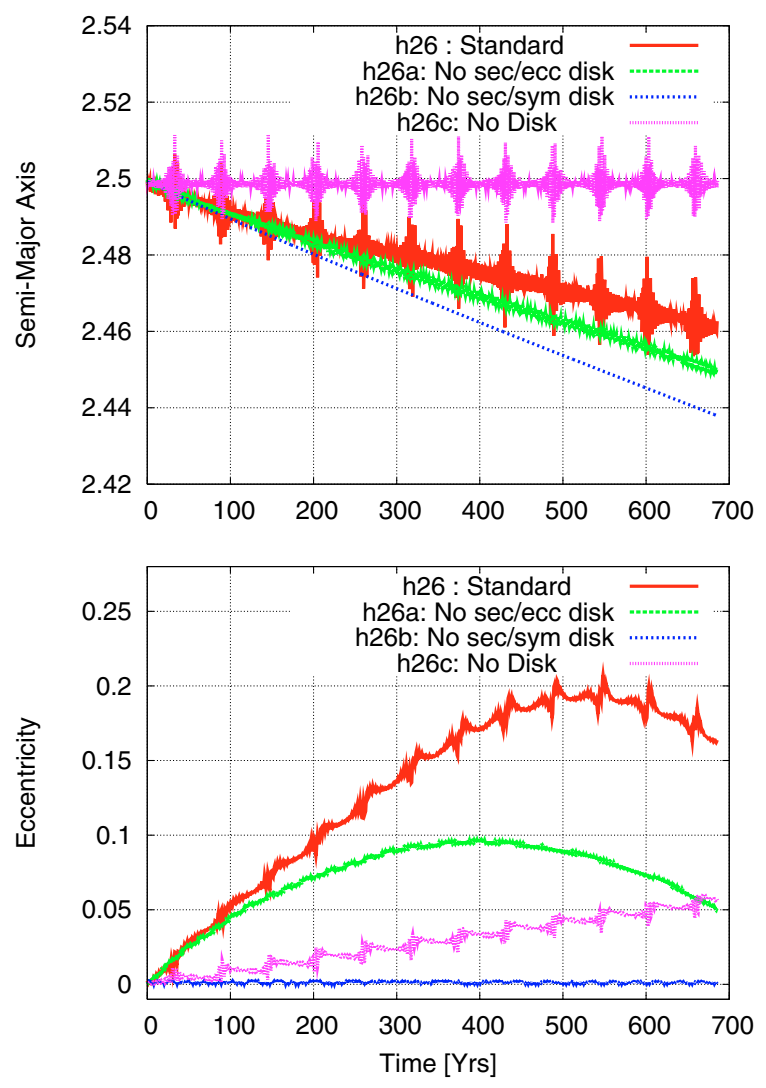

Fig. 6. Time evolution of the semi-major axis and eccentricity of the embedded planet for different physical set-ups, neglecting either the secondary (h26a, h26b) or the disc (h26c), see text for details.

for models (h26 and h26a) rises initially with the same speed but due to the fact that, without the secondary star, the disc slowly circularises, the model (h26a) falls behind the full model (which reaches $e_{\mathrm{p}}=0.2$ at $\left.t \approx 500\right)$ and $e_{\mathrm{p}}$ begins to drop off after a time of about $400 \mathrm{yrs}$ after insertion of the protoplanet. The maximum eccentricity reached for this reduced case (with no binary) is only $e_{\mathrm{p}}=0.1$. In the symmetrized disc case without secondary (h26b) the planet behaves as expected for single star discs, it displays inward migration (the fastest for all cases) and the eccentricity remains zero. The case with only the binary does not show any change in the semi-major axis $a_{\mathrm{p}}$ but rather a slow rise in $e_{\mathrm{p}}$. Additional test cases with similar set-up but different starting distances $a_{\mathrm{p}}=3.0$ and 3.5 AU display similar behaviour. For the largest initial distance, 3.5 AU, there are clear jumps in $e_{\mathrm{p}}$ evolution visible at each individual periapse. Hence, the rise of planetary eccentricity above the disc eccentricity for the full model is a combination of disc and binary effects.

To further study the consequences of the individual contributions we have displayed in Fig. 7 in addition to the standard model a model (h26a1) where just the dynamical (gravitational) effect of the secondary on the planet has been taken out. In contrast to the previous model (h26a) the secondary still acts here on the disc. In addition we display a model (h26t1) with a highly reduced planet mass of $5 \times 10^{-15} M_{\text {Earth }}$. The migration rate of the first two models is very similar while reduced (nearly zero) for the latter due to the smaller mass. The eccentricity evolution is initially very similar for all 3 models, while at later times the test particle simulation displays the largest planetary eccentricity growth and the model without the secondary the smallest. We suspect that the faster growth of $e_{\mathrm{p}}(t)$ for the test particle is a result of the vanishing migration and consequently larger
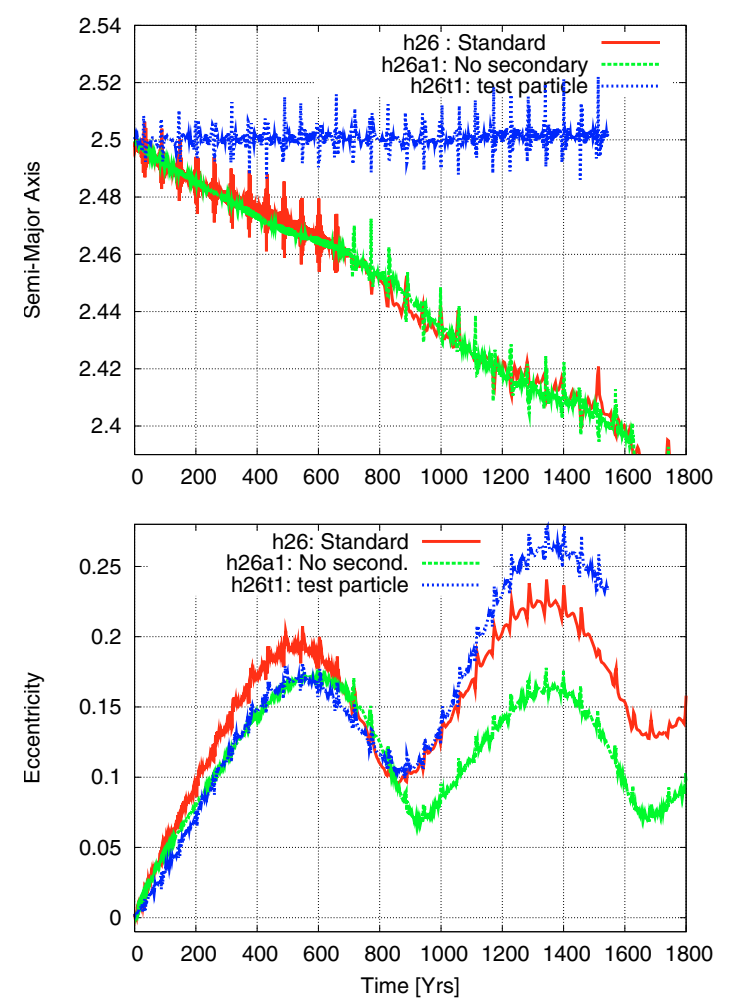

Fig. 7. Time evolution of the semi-major axis and eccentricity of the embedded planet for different physical set-ups. Here, the standard case (h26), a modified model where the dynamical influence of the secondary on the planet has been switched off (h26a1), and a model (h26t1) with a highly reduced planet mass (test particle with $5 \times 10^{-15} M_{\text {Earth }}$ ) are displayed.

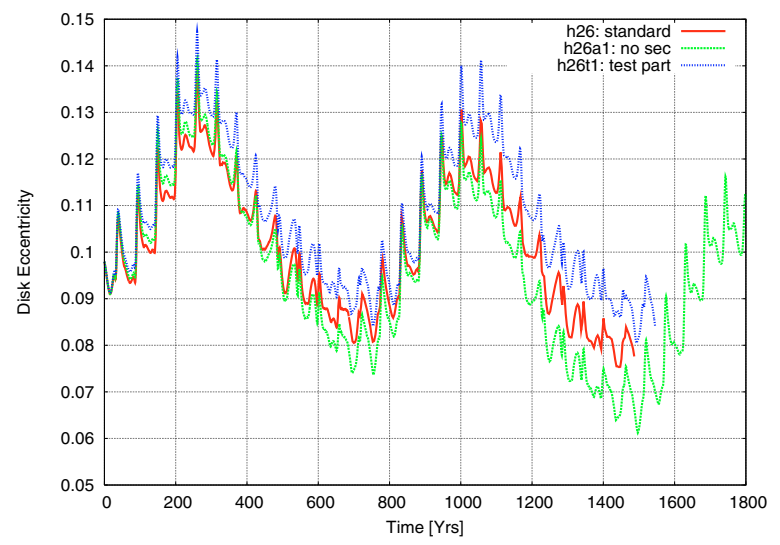

Fig. 8. Time evolution of the averaged disc eccentricity for three different physical set-ups. The standard case (h26), a modified model where the dynamical influence of the secondary on the planet has been switched off (h26a1), and a model (h26t1) with a highly reduced planet mass (test particle with $5 \times 10^{-15} M_{\text {Earth }}$ ) are displayed.

influence of the binary. In an additional plot (Fig. 8) the evolution of the averaged disc eccentricity for these models is displayed. In the very small test particle case (model h26t1) the disc eccentricity is only influenced by the binary and the $e_{\text {disc }}(t)$ evolution is a direct continuation of the initialisation process (refer to Fig. 4). From Figs. 7 and 8 it is apparent that there exists a phaseshift between the eccentricity of the disc and that of the planet. As demonstrated above (cf. Fig. 4) the variation of $e_{\text {disc }}(t)$ is correlated with the precession rate of the disc, and is due largely to the secular disc-binary interaction. The planetary 

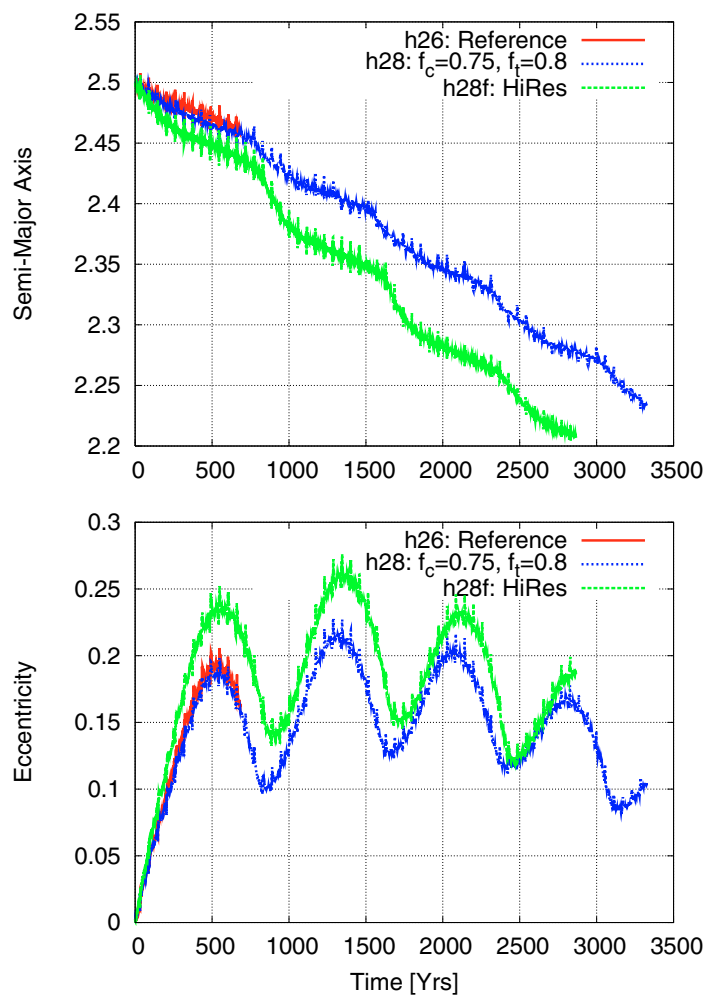

Fig. 9. Time evolution of the semi-major axis and eccentricity of the embedded planet for different numerical set-ups. For the reference case (h26) we used for the Courant number and force cutoff $f_{\mathrm{c}}=0.5$ and $r_{t}=1.0$, respectively. The second model (h28: dark dashed line, run only until $t \approx 700$ yrs) used $f_{\mathrm{c}}=0.75, r_{t}=0.8$, which has also been used for the last model (h28f: light dashed) that has a grid resolution of $500 \times 500$.

eccentricity on the other hand is driven primarily by secular interaction with the disc (as shown above), and the phaseshift between disc and planet eccentricity is caused by the shift in disc and planet longitudes of pericentre induced by their relative precession (see Fig. 11).

The two other models demonstrate that the disc eccentricity is clearly influenced by the presence of even a small mass planet of $36 M_{\text {Earth. In the case where the influence of the secondary has }}$ been switched off the planet's eccentricity is smaller as is the disc eccentricity. These results demonstrate that the combined evolution of planet and disc properties are rather intricately related in the case of binary stars. Hence, the evolution of the planet in this situation is more complicated in contrast to a planet embedded in an eccentric disc without a secondary star (see Papaloizou 2002), and not directly comparable.

To test the influence of numerical issues we perform for the reference case (h26) additional simulations with different setups. Among the purely numerical parameters altered are: the time step length as given through the Courant number $f_{c}$, the force cutoff radius $r_{t}$ as described in Sect. 2.4, and the grid resolution. The reference case in this section has been calculated with $f_{\mathrm{c}}=0.5, r_{t}=1.0$ and a resolution of $300 \times 300$. As displayed in Fig. 9, a change in the time-step and force cutoff to $f_{\mathrm{c}}=0.75, r_{t}=0.8$ (dark dotted curve until $t=3300$ ) has only a marginal influence. A change of resolution, here to $N_{\mathrm{r}} \times N_{\varphi}=500 \times 500$ leads to a faster migration rate $(30-$ $40 \%$ ) and slightly larger eccentricities. The qualitative behaviour (inward migration and saturation of eccentricity) is not changed, however. Given these encouraging results, we use for
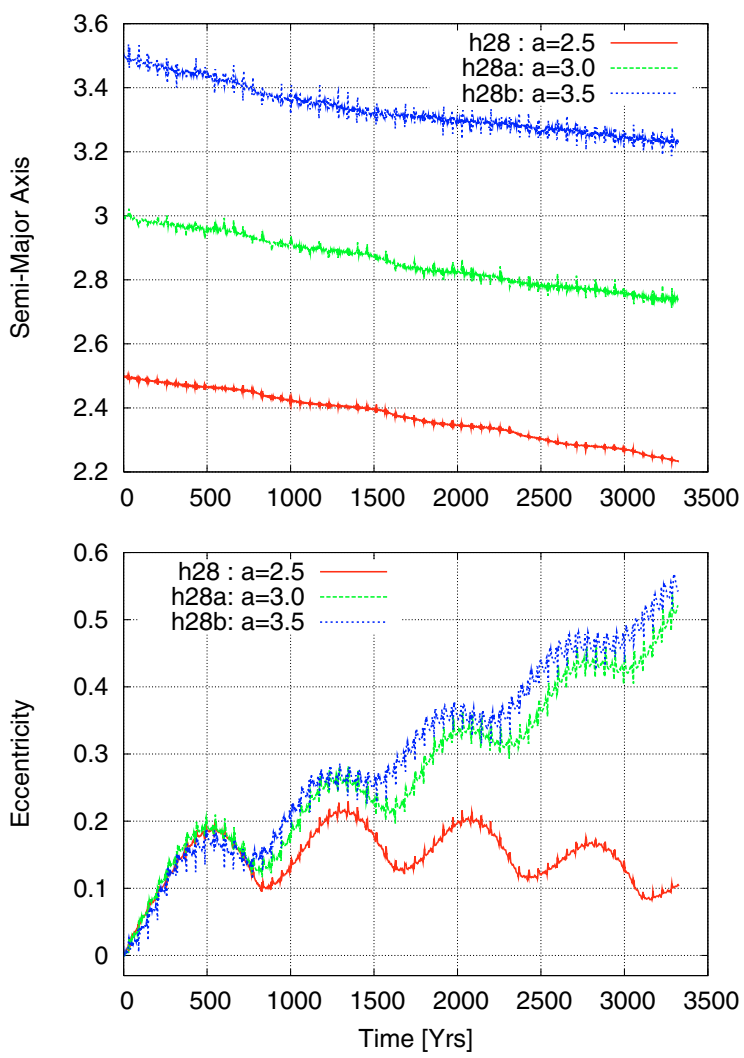

Fig. 10. Time evolution of the semi-major axis and eccentricity of a nonaccreting planet embedded planet for three different starting locations.

computational reasons the reference resolution of $300 \times 300$ and $f_{\mathrm{c}}=0.75, r_{t}=0.8$ in our subsequent models.

\subsection{Models without accretion onto planet}

Planetary cores form in the outer cooler regions of protoplanetary discs beyond the so-called ice-line. In a binary star system the outer disc is affected most by the secondary, and to find possible restrictions on the planet forming regions in the disc it is important to analyse the evolution of cores near the outer parts of the disc. To study the effect of initial position we start our embryos at 3 different locations in the disc 2.5, 3.0 and 3.5 AU always on a circular orbit, and choose again non-accreting cores. Because the initial characteristic growth time of the cores may be long, even in comparison to the orbital period of the binary these sets of runs constitute a test suite to estimate the orbital evolution of protoplanets in the disc. The results for the semi-major axis and eccentricity evolution of the planet are displayed in Fig. 10, where the only difference in the three cases is the release distance $(2.5,3.0$ and $3.5 \mathrm{AU})$ of the planet. From all three locations the planets migrate inwards at approximately the same rate with the tendency for a slowdown for the two outer cases. This inward migration of the outer planets which are mainly orbiting outside the disc is due the the planet disc interaction near planetary periastron where the planet moves faster through the disc and induces a negative torque and power (energy loss) (Cresswell et al. 2007). However, the different initial starting radii lead to a very different eccentricity evolution. While only the innermost planet (starting at 2.5 AU) shows a finite eccentricity evolution the two outer cases display a very strong increase in their eccentricity beyond $e_{\mathrm{p}}=0.5$ after about 55 binary orbits. Clearly the strongly disturbed disc in the outer regions at around $4 \mathrm{AU}$ 


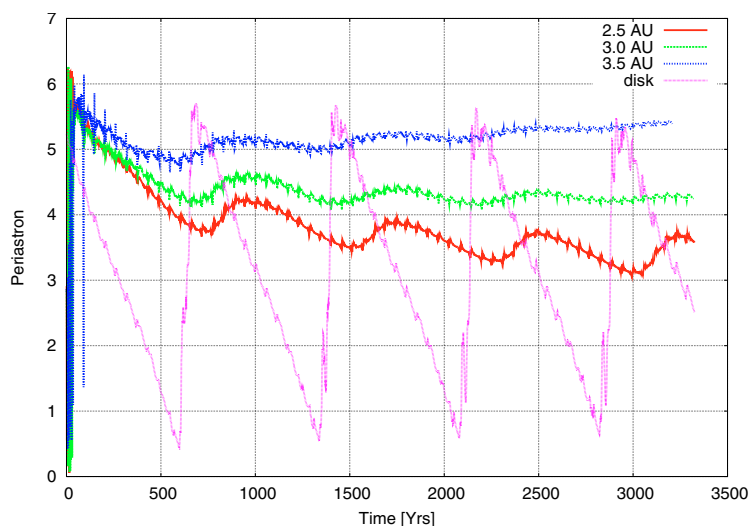

Fig. 11. Time evolution of the argument of pericentre (periastron) for an embedded planet starting at 3 different locations and the protoplanetary disc.

significantly perturbs the orbits of the protoplanet and does not allow for small equilibrium eccentricity. While the initial faster rise of $e_{\mathrm{p}}$ appears to be caused primarily by the disc, the longterm evolution to very high eccentricities is most likely due to the combined action of the binary and the eccentric disc. A test simulation (for an initial semi-major axis $a_{\mathrm{p}}=3.0 \mathrm{AU}$ ) where the gravitational action on the planet due to the secondary has been switched off did not display any large eccentricity growth but a steady decline. On the other hand, starting the planets with high eccentricity (from $a_{\mathrm{p}}=3.0 \mathrm{AU}$ ) in a pure 3-body problem does also not lead necessarily to a growth of eccentricity but rather to an oscillatory behaviour. So we may conclude that in the present situation it is the combined action of an eccentric, periodically perturbed disc and the influence of an eccentric binary star.

As the planets move on non-circular orbits in an eccentric disc and binary a temporal change of the apsidal line may be expected. In Fig. 11 the evolution of the argument of pericentre of the planets and the disc are plotted versus time. The innermost planet has on average a periastron angle of about $200 \mathrm{deg}$ while the outer planets have a larger angle. For the binary the angle of periastron lies fixed at 0 degrees, and the disc is slowly precessing retrograde as pointed out above and indicated in Fig. 11 by the tightly dashed line. The influence of the disc on the planets is visible by the slight oscillations of the periastron angle about the mean with the same period as the oscillations in the eccentricity. The innermost planet has approximately a phase shift of $180 \mathrm{deg}$ with respect to the binary and is nearly in an anti-symmetric state while the other planets are lagging behind this configuration.

\subsection{Models with mass accretion onto planet}

To make the scenario more realistic we add now the option of mass accretion onto the protoplanet. To do so, we assume that initially the planets grew during a long time to their present mass and enter now the more rapid gas accretion phase. Numerically we continue the previous simulations for the three initial radii at a time $t=2500 \mathrm{yrs}$. The evolution of the orbital elements of the planets are displayed in Fig. 12 where the time offset has been reset after the turn-on of mass accretion. The thinner lines (until $t \approx 3000$ ) are the models with accretion switched on at a low rate using an efficiency of $f_{\text {acc }}=3 \times 10^{-2}$. The symbols refer to the final phase of the models without accretion. While the eccentricities of the outer planets continue to rise to very high values despite a slow inward migration, the inner planet's orbit (starting at $a_{\mathrm{p}}=2.3 \mathrm{AU}$ ) begins to circularise while the inward
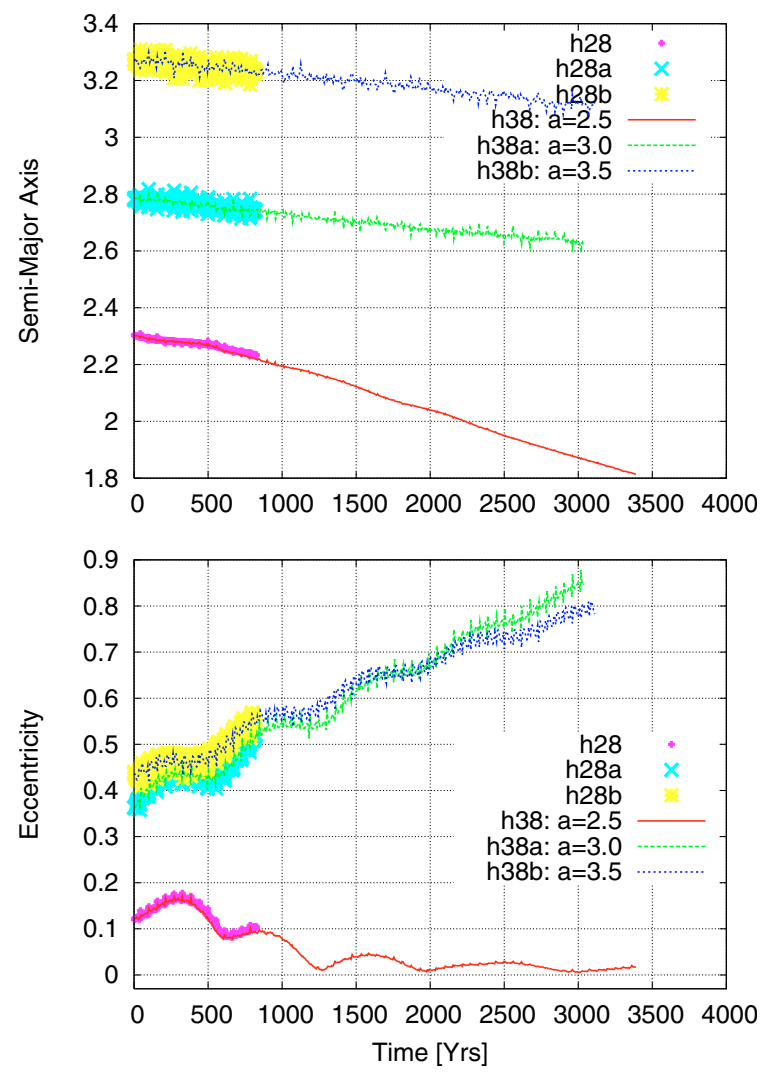

Fig. 12. Time evolution of the semi-major axis and eccentricity for 3 accreting protoplanets starting at 3 different locations in the disc. The symbols refer to the non-accreting models of Fig. 10 time-shifted by $2500 \mathrm{yrs}$, while the lines refer to restarted models with accretion switched on.

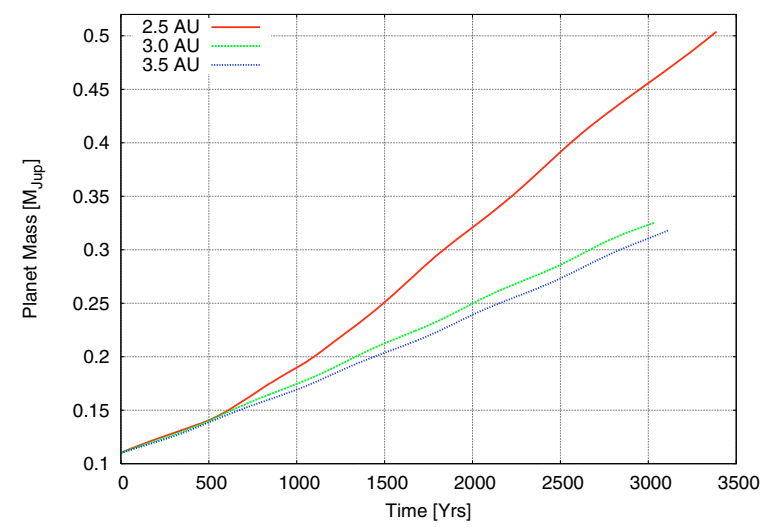

Fig. 13. Mass of the planet vs. time for accreting models for 3 different initial locations of the planet.

migration speed increases. The higher eccentricities of the outer planets can probably only be maintained in a stable way because of some eccentricity damping action of the disc during their periastron passage. The planetary mass evolution for the three cases is displayed in Fig. 13. The innermost planet has reached about half a Jupiter mass after 3400 yrs while the other two grow at a smaller rate and have reached about $0.3 M_{\text {Jup }}$ after 3000 yrs. Note that these labels refer to the initial positions of the planets that were first evolved without mass accretion and then continued with $f_{\text {acc }} \neq 0$ as described above. From the simulations it seems to be clear that it may be difficult to grow a planet at too large a distance from the primary star. For our chosen binary 

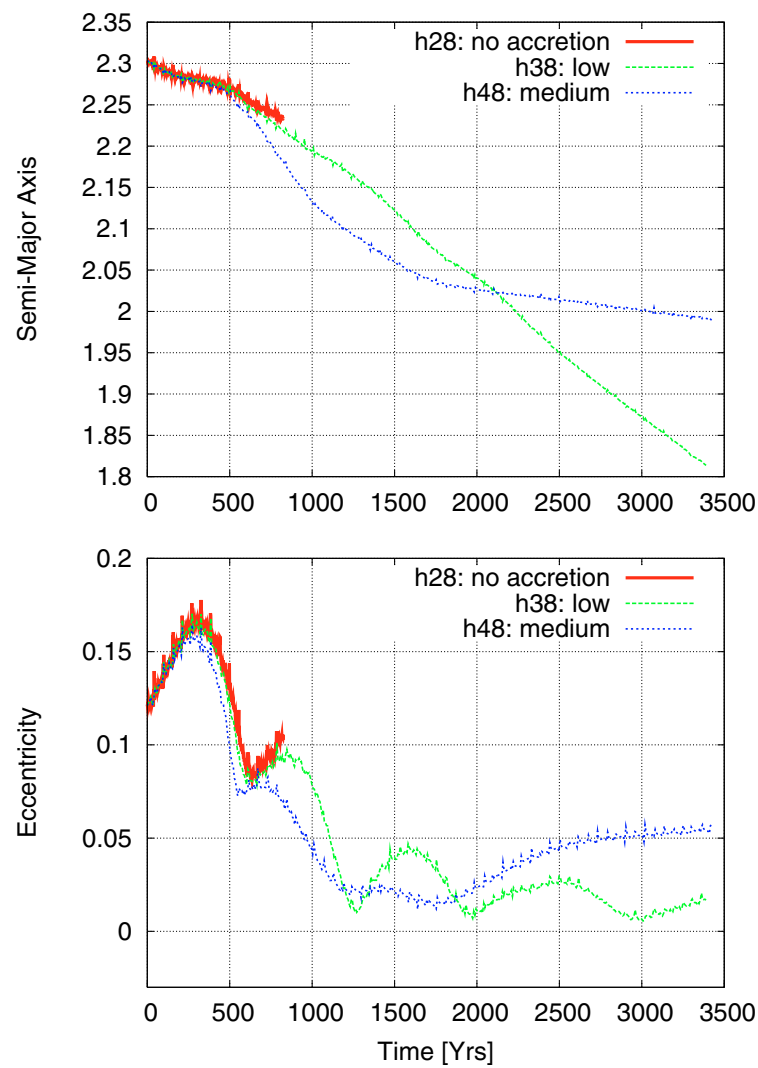

Fig. 14. Time evolution of the semi-major axis and eccentricity of 3 accreting protoplanets starting at the same initial condition with different mass accretion rates.

parameter the borderline lies around 2.7 AU, which has been verified by additional simulations with different starting distances that are not shown here. This particular transition radius of about 2.7 AU depends on the chosen parameters for the disc viscosity and temperature. Larger values can possibly extend this transition radius slightly while smaller values will reduce it. Varying the disc mass will change the viscosity and temperature of the disc, and we expect this to influence the transition radius, too. It is beyond the scope of the present to paper to perform detailed parameter studies on these issues. From the results displayed in Fig. 13 it appears that mass accretion onto the planet does not alter the orbital evolution of the planet significantly.

To further explore parameter space we used the starting condition $a_{\mathrm{p}}=2.5$ and performed one additional run with a three times higher accretion rate onto the planet $\left(f_{\text {acc }}=10^{-1}\right.$, denoted "medium" in the figures). The results of this new model with the previous no accretion and low accretion models are shown in Fig. 14. The results for the orbital elements of the planet in Fig. 14 show that the models with no and low accretion behave similarly. The planet continues to migrate inward while decreasing its eccentricity. The model with the higher accretion rate migrates faster at first, and slows down near the end, while the eccentricity settles to about $e_{\mathrm{p}}=0.05$. This reduction in the migration rate is a consequence of the increasing mass of the planet. For this higher accretion rate the planet's mass increases to nearly $1.7 M_{\text {jup }}$ after 3300 years (see Fig. 15). Since the disc mass is reduced by the same amount the driving agent of the planet is lost and the speed of migration reduced. This model with the "medium" accretion rate settles to a final state which is quite close to the observed value for the $\gamma$ Cep system.

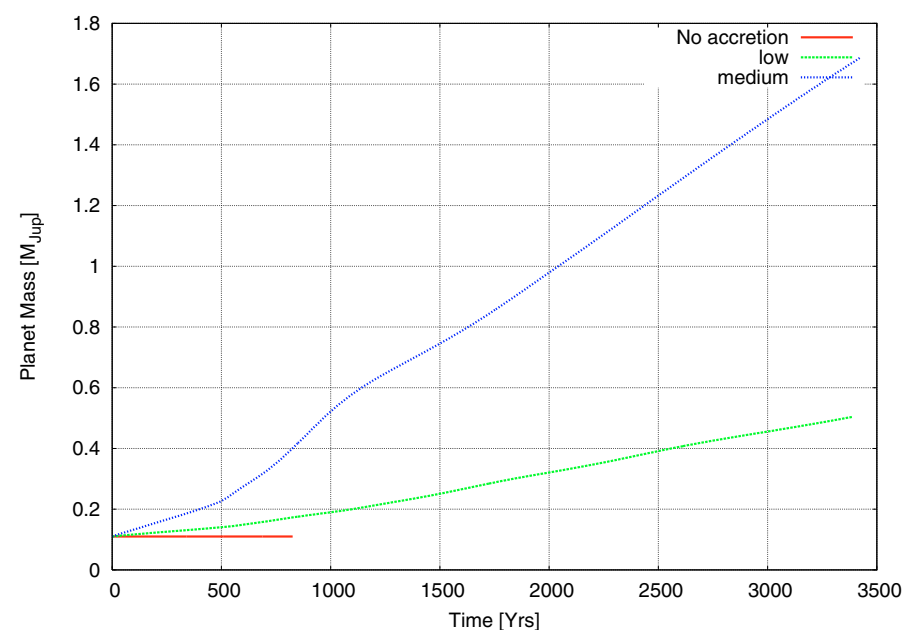

Fig. 15. Mass of the planet vs. time for accreting models starting at $a_{\mathrm{p}}=$ 2.5 AU for 3 different accretion rates.

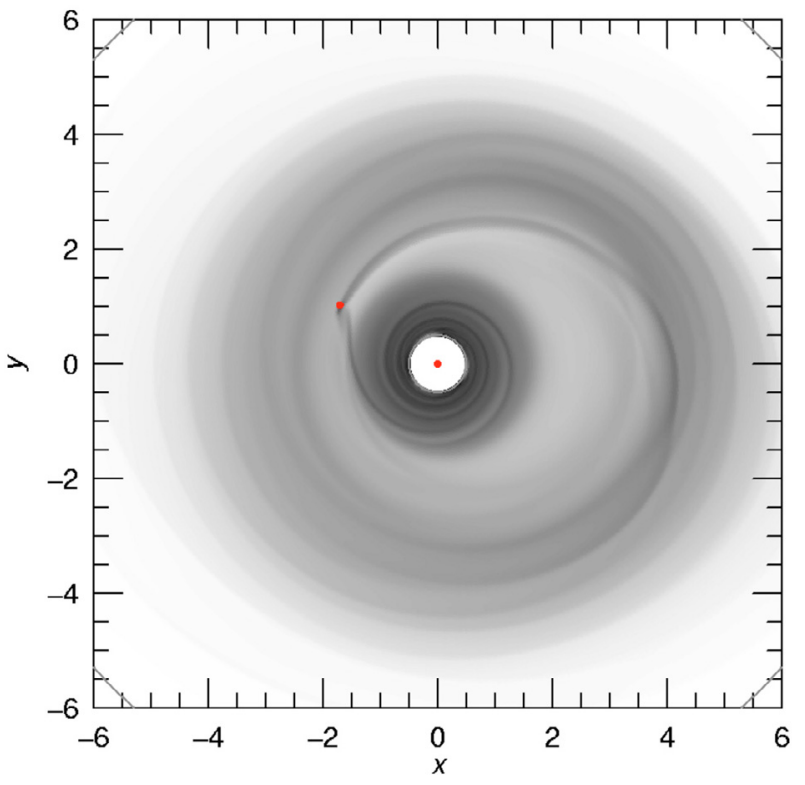

Fig. 16. Grayscale plot of the two dimensional density distribution of the medium accretion model ( $\mathrm{h} 48$ ) at time $3125 \mathrm{yrs}$. The shading is scaled $\propto \Sigma^{1 / 4}$ between $4.8 \times 10^{-4}$ (white) and $2400 \mathrm{~g} / \mathrm{cm}^{2}$ (black). The location of the planet is marked by the small circle.

A massive embedded planet will open a gap in standard circular discs, and it is interesting to analyse this effect within the present context. In Fig. 16 we display the two-dimensional density distribution $\Sigma(r, \varphi)$ in the disc at a time $3125 \mathrm{yrs}$ for the medium accretion model. At this time the planet has reached approximately a mass of $1.5 M_{\text {Jup }}$. From the plot it seems that the disc inside the planetary orbit is apparently more circular than outside. This is confirmed by the corresponding onedimensional radial distribution of the azimuthally averaged density and eccentricity of the disc at the same time. The gap is somewhat weaker than in circular discs primarily due to the periodic disturbance of the secondary that tends to sweep material into the cleared region around the planet. Due to the shallower gap the planet is able to continue mass accretion from its surroundings more easily compared to a planet on a circular orbit in a single star system. The inner disc clearly has a smaller eccentricity than the outer parts (compare this to Fig. 3). The presence of the planet represents a barrier for the (spiral) wave induced 

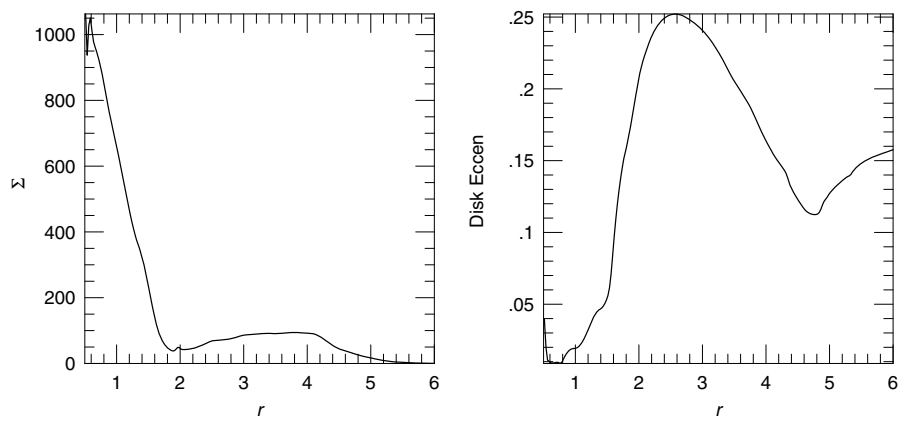

Fig. 17. Azimuthally averaged density (in $\mathrm{g} / \mathrm{cm}^{2}$ ) and eccentricity of the disc for the medium accretion model at a time of $3125 \mathrm{yrs}$.

by the binary which consequently cannot propagate into the inner parts of the disc.

\section{Conclusions and summary}

In the present work we have concentrated on the planetary formation in the system $\gamma$ Cep which places, due to its binary parameter of $a_{\text {bin }}=20 \mathrm{AU}$ and $e_{\text {bin }}=0.41$, severe constraints on the formation process. We investigated an intermediate phase in the planet formation process within the so-called core formation scenario, i.e. we did not attempt to model the formation of planetesimals in this system but rather concentrated on a later phase when protoplanetary cores have already formed and begin their rapid gas accretion phase.

Before embedding the protoplanets in the disc, we have first brought the system into equilibrium by performing simulations with no embedded protoplanets. This initialisation phase takes about 100 binary orbits after which the disc structure has settled to a new quasi-equilibrium configuration with a truncated disc. Interestingly, the accretion disc around the primary reaches an average eccentricity of about $e_{\text {disc }}=0.12$ and shows a coherent retrograde precession, in agreement with the findings of Paardekooper et al. (2008). A detailed parameter study of this interesting behaviour on its own is beyond the present analysis and will be presented elsewhere. During one binary orbit the eccentric secondary disturbs the disc periodically and induces significant non-axisymmetric perturbations in the disc which decay due to viscosity during binary apoapse.

As suspected these perturbations of the disc, in particular the periodic creation of strong tidally induced spiral density waves and the creation of an eccentric disc, lead to non-negligible effect on the planetary orbital elements. While embryos placed in the disc at different initial distances from the primary star continue to migrate inward at approximately the same rate, the eccentricity evolution is markedly different for the individual cases. If the initial distance is beyond about $a \gtrsim 2.7$ AU the eccentricity of the embryo continues to rise to very high values and, apparently only due to the damping action of the disc, the orbits remains bound. The excitation mechanism of the eccentricity is the combined action of the binary and the perturbed disc (i.e. there is a long term secular interaction due to the disc being eccentric). Test simulations where individual components of the system have been switched off and on have made it clear that it is indeed the combination of effects (eccentric binary and the eccentric, periodically perturbed disc) that leads to the high planetary eccentricities in the system. Once $e_{\mathrm{p}}$ has grown to sufficiently large values the planetary orbit may reach the stability limit as given for example by the pure 3-body simulations for $\gamma$ Cep (see Fig. 2 in Turrini et al. 2004).

A low mass, non accreting planet embedded in an the eccentric disc experienced substantial growth in eccentricity (see Fig. 10). This has clear implications for the accretion of planetesimals because their velocity dispersion may become very large due to this effect. Thébault et al. (2004) examined the evolution of planetesimal orbits under the influence of the binary companion and aerodynamical gas drag. They concluded that accretion of planetesimals would occur in the shear dominated regime because orbital alignment was maintained due to the gas drag. This work, however, did not include the effects of an eccentric disc, and so it remains unclear whether planetesimal orbits will remain aligned. Recently, this issue has been addressed by Kley \& Nelson (2007) and Paardekooper et al. (2008) who find that the inclusion of the dynamical evolution of the disc may render the planetesimal formation more difficult, but this topic certainly deserves more consideration.

The most up to date observational data suggest the following parameters for the planet in the $\gamma$ Cep system: $a_{\mathrm{p}} \simeq 2.044$, $e_{\mathrm{p}} \simeq 0.115$ and $m_{\mathrm{p}} \sin i \simeq 1.60 M_{\text {Jup }}$. If this planet formed according to the core instability model, then an important issue is the survival of the planetary core before gas accretion occurs. Figure 10 has shown that the non-accreting, low mass planet undergoes quite rapid inward migration. The migration, however, is modulated by the eccentricity of the planet, such that at high eccentricity phases the migration rate decreases. It is possible that longer run times will show an essential stalling of this migration if the planet eccentricity grows beyond its final value of $e_{\mathrm{p}} \simeq 0.3$. The inclusion of a better approximation to the physical state of the disc, eg. radiative cooling, may help to slow down this initial phase, as new results indicate that in case of radiative discs migration is slowed down or even reversed for protoplanetary cores (Paardekooper \& Mellema 2008; Baruteau \& Masset 2008).

Once gas accretion is switched on, it is clear that a disc mass of about 3 Jupiter masses, where the outer disc radius is tidally truncated at $r \simeq 5 \mathrm{AU}$, will be sufficient to grow a planet that is close to the minimum observed mass of $m_{\mathrm{p}} \sin i \simeq 2.044 M_{\mathrm{Jup}}$. It is also clear that we can construct a model in which a low mass planet growing from an initially circular orbit can achieve a final mass of $m_{\mathrm{p}} \simeq 2 M_{\mathrm{Jup}}$, and have a final eccentricity of $e_{\mathrm{p}} \simeq 0.1$ as required.

A final comment relates to the final mass of the planet. Our simulations suggest that a disc mass of about 3 Jupiter masses will be enough to form a gas giant of the required minimum mass. A future test of the mode by which the planet in $\gamma$ Cep formed (gravitational instability versus core accretion) will be determination of its actual mass. We suspect that a disc that is massive enough to form a planet through gravitational instability will lead to a planet whose final mass is substantially larger than the minimum value observed.

Acknowledgements. Very useful discussions with Dr. Paardekooper at the DAMTP in Cambridge are gratefully acknowledged. The work was sponsored in parts by the EC-RTN Network The Origin of Planetary Systems under grant HPRN-CT-2002-00308, and by grant KL 650/6 of the German Research Foundation (DFG). Some of the simulations conducted as part of this project were performed on the QMUL High Performance Computing facility funded through the SRIF initiative.

\section{References}

Baruteau, C., \& Masset, F. 2008, ApJ, 672, 1054

Boss, A. P. 1998, BAAS, 30, 1057

Boss, A. P. 2006, ApJ, 641, 1148 
Chambers, J. E., Quintana, E. V., Duncan, M. J., \& Lissauer, J. J. 2002, AJ, 123, 2884

Cresswell, P., Dirksen, G., Kley, W., \& Nelson, R. P. 2007, A\&A, 473, 329

Crida, A., Sándor, Z., \& Kley, W. 2008, A\&A, 483, 325

Desidera, S., \& Barbieri, M. 2007, A\&A, 462, 345

Dvorak, R. 1986, A\&A, 167, 379

Dvorak, R., Pilat-Lohinger, E., Bois, E., et al. 2004, in Rev. Mex. Astron. Astrofis. Conf. Ser., ed. C. Allen, \& C. Scarfe, 21, 222

Eggenberger, A., Udry, S., \& Mayor, M. 2004, A\&A, 417, 353

Eggenberger, A., Udry, S., Mazeh, T., Segal, Y., \& Mayor, M. 2007, A\&A, 466, 1179

Haghighipour, N. 2006, ApJ, 644, 543

Hatzes, A. P., Cochran, W. D., Endl, M., et al. 2003, ApJ, 599, 1383

Heppenheimer, T. A. 1974, Icarus, 22, 436

Heppenheimer, T. A. 1978, A\&A, 65, 421

Jang-Condell, H. 2007, ApJ, 654, 641

Kley, W. 1989, A\&A, 208, 98

Kley, W. 1999, MNRAS, 303, 696

Kley, W. 2000, in IAU Symp. 200, ed. H. Zinnecker, \& R. Mathieu, 211

Kley, W., \& Nelson, R. 2007, ArXiv e-prints, [arXiv:0705.3421]

Konacki, M. 2005a, Nature, 436, 230

Konacki, M. 2005b, American Astronomical Society Meeting Abstracts, 207

Lissauer, J. J., Quintana, E. V., Chambers, J. E., Duncan, M. J., \& Adams, F. C. 2004, in Rev. Mex. Astron. Astrofis. Conf. Ser., 99

Lubow, S. H. 1991, ApJ, 381, 259

Masset, F. 2000, A\&AS, 141, 165

Mugrauer, M., Neuhäuser, R., Seifahrt, A., Mazeh, T., \& Guenther, E. 2005, A\&A, 440, 1051

Muterspaugh, M. W., 2005, PTI: Palomar Testbed Interferometer Collaboration, \& PHASES Team. American Astronomical Society Meeting Abstracts, 207

Nelson, A. F. 2000, ApJ, 537, L65
Nelson, R. P. 2003, MNRAS, 345, 233

Nelson, R. P., Papaloizou, J. C. B., Masset, F. S., \& Kley, W. 2000, MNRAS, 318, 18

Neuhäuser, R., Mugrauer, M., Fukagawa, M., Torres, G., \& Schmidt, T. 2007, A\&A, 462, 777

Paardekooper, S.-J., \& Mellema, G. 2008, A\&A, 478, 245

Paardekooper, S.-J., Thébault, P., \& Mellema, G. 2008, MNRAS, 386, 973

Papaloizou, J. C. B. 2002, A\&A, 388, 615

Papaloizou, J. C. B. 2005, A\&A, 432, 757

Papaloizou, J. C. B., \& Nelson, R. P. 2005, A\&A, 433, 247

Pfahl, E. 2005, ApJ, 635, L89

Pierens, A., \& Nelson, R. P. 2007, A\&A, 472, 993

Pierens, A., \& Nelson, R. P. 2008a, A\&A, 482, 333

Pierens, A., \& Nelson, R. P. 2008b, A\&A, 478, 939

Portegies Zwart, S. F., \& McMillan, S. L. W. 2005, ApJ, 633, L141

Takeda, G., \& Rasio, F. A. 2005, ApJ, 627, 1001

Tanaka, H., Takeuchi, T., \& Ward, W. R. 2002, ApJ, 565, 1257

Thébault, P., Marzari, F., Scholl, H., Turrini, D., \& Barbieri, M. 2004, A\&A, 427, 1097

Thébault, P., Marzari, F., \& Scholl, H. 2006, Icarus, 183, 193

Turrini, D., Barbieri, M., Marzari, F., \& Tricarico, P. 2004, Mem. Soc. Astron. It. Suppl., 5, 127

Turrini, D., Barbieri, M., Marzari, F., Thebault, P., \& Tricarico, P. 2005, Mem. Soc. Astron. It. Suppl., 6, 172

van Leer, B. 1977, J. Comput. Phys., 23, 276

Verrier, P. E., \& Evans, N. W. 2006, MNRAS, 368, 1599

Whitmire, D. P., Matese, J. J., Criswell, L., \& Mikkola, S. 1998, Icarus, 132, 196 Wu, Y., \& Murray, N. 2003, ApJ, 589, 605

Wu, Y., Murray, N. W., \& Ramsahai, J. M. 2007, ApJ, 670, 820

Ziegler, U. 1998, Comp. Phys. Commun., 109, 111

Ziegler, U., \& Yorke, H. 1997, Comp. Phys. Commun., 101, 54 